\title{
PRIMARY IDEALS AND VALUATION IDEALS. II
}

\author{
BY \\ ROBERT GILMER( $\left.{ }^{1}\right)$ AND WILLIAM HEINZER
}

Introduction. Let $D$ be an integral domain with identity. In [2], Gilmer and Ohm considered the problem of characterizing domains $D$ such that the set $\mathscr{Q}(D)$ of primary ideals of $D$ is a subset of the set $\mathscr{V}(D)$ of valuation ideals of $D$. If the ascending chain condition (a.c.c.) for prime ideals holds in $D$, then $\mathscr{Q}(D) \subseteq \mathscr{V}(D)$ if and only if $D$ is a Prüfer domain; a domain in which primary ideals are valuation ideals need not be Prüfer if the assumption concerning the a.c.c. for prime ideals is dropped (see Theorem 3.8 and $\S 5$ of [2]). In case a.c.c. for prime ideals does not hold in $D$, Gilmer and Ohm left open the question as to when primary ideals of $D$ are valuation ideals.

In [1], Gilmer showed that the question as to whether $\mathscr{Q}(D) \subseteq \mathscr{V}(D)$ or not is closely related to the structure of the set of prime ideals of $D$. Before mentioning these results and their relation to this paper, we introduce some terminology. Let $R$ be a commutative ring with identity, let $P$ be a prime ideal of $R$, and let $\left\{Q_{\alpha}\right\}$ be the set of $P$-primary ideals of $R$. We consider the following conditions:

I. $\left\{Q_{\alpha}\right\}$ is linearly ordered under $\subseteq$.

II. $M=\bigcap_{\alpha} Q_{\alpha}$ is a prime ideal.

III. There are no prime ideals of $R$ properly between $M$ and $P$.

IV. If $P_{1}$ is any prime ideal of $R$ properly contained in $P$, then $P_{1} \subseteq M$.

Following [1], we say $P$ is an $S$-ideal if I, II, and IV hold. It is clear that IV implies III. If I-III hold, we say $P$ is a weak $S$-ideal. $R$ is an $S$-ring if each prime ideal of $R$ is an $S$-ideal; weak $S$-ring is defined analogously. Corollary 2.4 of [1] shows that if $D$ is an $S$-domain, then $\mathscr{Q}(D) \subseteq \mathscr{V}(D)$. The proof of Corollary 2.4 does, in fact, show that in a weak $S$-domain primary ideals are valuation ideals. The status of the converse of Corollary 2.4 was considered in [1], but was not determined.

In $\S 3$ we prove that for $P$ prime in $D$, each $P$-primary ideal is a valuation ideal if and only if conditions I and II hold. We thereby obtain what we feel is a satisfactory characterization of domains in which primary ideals are valuation ideals. To resolve the questions of whether the condition $\mathscr{Q}(D) \subseteq \mathscr{V}(D)$ implies $D$ is an $S$ domain or a weak $S$-domain we need only determine in the global case whether condition IV or condition III depends upon I and II. These questions are answered in §5. Example 5.8 shows III does not depend on I and II and hence IV does not

Presented to the Society, November 11, 1966; received by the editors July 19, 1966.

(1) During the writing of this paper, the first author received support as an Alfred P. Sloan fellow. 
depend on I and II. Example 5.9 shows IV is independent of I-III-that is, a weak $S$-domain need not be an $S$-domain. We begin in $\$ 2$ by considering a prime ideal $\boldsymbol{P}$ of a commutative ring with identity such that the set of $\boldsymbol{P}$-primary ideals is chained under $\subseteq$.

All rings considered in this paper are assumed to be commutative and we assume that each integral domain considered has an identity. The terminology used is that of Zariski-Samuel [6], [7]. We shall make frequent use of the material from [6] on quotient rings of, in our case, an integral domain. We shall also use the fact that in considering $v$-ideals of a domain $D$, where $v$ is a valuation nonnegative on $D$, there is no loss of generality in assuming that $D$ and the valuation ring of $v$ have the same quotient field [7, p. 340]. While we feel that a reader can understand most of the results of this paper without having read [2], it is unlikely that he can appreciate its results independent of [1] and [2].

2. Linearly ordered systems of primary ideals. Throughout this section $P$ denotes a prime ideal of $R$, a commutative ring with identity, and $\left\{Q_{\alpha}\right\}$ denotes the set of $P$-primary ideals. We shall assume that the set $\left\{Q_{\alpha}\right\}$ is linearly ordered under $\subseteq$; we denote by $M$ the intersection of the $Q_{\alpha}$ 's.

2.1. Lemma. If $\left(Q_{\beta}\right\}$ is a subset of $\left\{Q_{\alpha}\right\}$ such that $B=\bigcap_{\beta} Q_{\beta} \supset M$, then $\sqrt{ } B=P$. Equivalently, if $\sqrt{ } B \subset P$, then $B=M$.

Proof. Since $B \supset M$, there exists a $P$-primary ideal $Q$ such that $B \nsubseteq Q$. Thus $Q_{\beta} \ddagger Q$ for any $\beta$ so that $Q \subseteq Q_{\beta}$ for each $\beta$, and consequently, $Q \subseteq \bigcap_{\beta} Q_{\beta}=B$. Hence $P=\sqrt{ } Q \subseteq \sqrt{ } B \subseteq P$.

We shall find it convenient in the results immediately following to consider the case when $P$ is maximal in $R$. We are able to relate these results to similar questions in the general case by observing that in the quotient ring $R_{P}, P^{e}$ is maximal and $\left\{Q_{\alpha}^{e}\right\}$, the set of $P^{e}$-primary ideals of $R_{P}$, is linearly ordered under $\subseteq$; here " $e$ " denotes extension with respect to $R_{P}[6, \mathrm{p} .218]$. Then we use known results concerning the relationship between the ideal theory of $R$ and that of $R_{P}[6, \mathrm{pp}$. 223-233].

2.2. Proposition. If $P$ is maximal in $R$, then for any $P$-primary ideal $Q$, either $P Q=Q$ or $P Q \subset Q$ and each $P$-primary ideal properly contained in $Q$ is contained in $P Q$.

Proof. We suppose $P Q \subset Q$. Then $Q / P Q$ is an $R$-module and $P$ is contained in the annihilator of this module over $R$. Hence the structure of $Q / P Q$ as an $R$-module is the same as its structure as an $R / P$-module - that is, as a vector space over $R / P$. The submodules of $Q / P Q$ correspond to the ideals $A$ of $R$ such that $P Q \subseteq A \subseteq Q$. Any such ideal $A$ has radical $P$ and must therefore be $P$-primary since $P$ is maximal in $R\left[6\right.$, p. 153]. Thus, by hypothesis on $\left\{Q_{\alpha}\right\}$, the subspaces of the vector space $Q / P Q$ over $R / P$ are linearly ordered so that $Q / P Q$ is one dimensional. It follows that there are no ideals of $R$ properly between $P Q$ and $Q$. Since each $P$-primary 
ideal $Q^{\prime}$ properly contained in $Q$ compares with $P Q$ under $\subseteq$, it follows that each such $Q^{\prime}$ must be contained in $P Q$. Q.E.D.

Before proceeding further we introduce some notation. If $x \in R-M$ we denote by $B_{x}$ the intersection of all $P$-primary ideals which contain $x$ (if $x \notin P$, then $\left.B_{x}=R\right)$. If $x \in P-M$, Lemma 2.1 shows that $B_{x}$ is an ideal of $R$ having radical $P$. Hence if $P$ is maximal and if $x \in P-M$, then $B_{x}$ is the smallest $P$-primary ideal of $R$ containing $x$. For $P$ maximal we have for any $x \in R-M, B_{x}=\bigcap_{\alpha}\left(Q_{\alpha}+(x)\right)$.

2.3. Lemma. If $P$ is maximal in $R$ and if $x, y \in R-M$, then $B_{x} B_{y} \subseteq \bigcap_{\alpha}\left(Q_{\alpha}+(x y)\right)$. If $x y \notin M$, then $B_{x} B_{y}=B_{x y}$.

Proof. If $s \in B_{x}$ and $t \in B_{y}$, then for any $\alpha$ we have $s \in Q_{\alpha}+(x)$ and $t \in Q_{\alpha}+(y)$ so that $s t \in\left(Q_{\alpha}+(x)\right)\left(Q_{\alpha}+(y)\right) \subseteq Q_{\alpha}+(x y)$. Hence $s t \in \bigcap_{\alpha}\left(Q_{\alpha}+(x y)\right)$ and

as we wished to show.

$$
B_{x} B_{y} \subseteq \bigcap_{\alpha}\left(Q_{\alpha}+(x y)\right)
$$

If $x y \notin M$ then we have just shown that $B_{x} B_{y} \subseteq B_{x y}$. However $\sqrt{ }\left(B_{x} B_{y}\right)=P$ so that $B_{x} B_{y}$ is a $P$-primary ideal containing $x y$. Hence $B_{x y} \subseteq B_{x} B_{y}$. Q.E.D.

2.4. Proposition. If $P$ is maximal in $R$ and if $M$ does not have radical $P$, then $M$ is a prime ideal.

Proof. Let $x, y \in R-M$. We show $x y \in R-M$. If $x, y \in R-P$ it is clear that $x y \in R-M$. If $x y \in P$, Lemma 2.3 shows that $B_{x} B_{y} \subseteq \bigcap_{\alpha}\left(Q_{\alpha}+(x y)\right) \subseteq P$ so that $\sqrt{ }\left(\bigcap_{\alpha}\left(Q_{\alpha}+(x y)\right)\right)=P$. Because $\sqrt{ } M=\sqrt{ }\left(\bigcap_{\alpha} Q_{\alpha}\right) \subset P$ it follows that for some $\alpha$, $x y \notin Q_{\alpha}$. Therefore $x y \notin M$ and $M$ is a prime ideal. Q.E.D.

\subsection{Corollary. If $M$ does not have radical $P, M$ is a prime ideal.}

Proof. If $e$ and $c$ denote extension and contraction with respect to $R$ and $R_{P}$ (see [6, p. 218]) we first show that $M=M^{e c} . \subseteq$ is clear. If $x \in M^{e c}$ there is an element $y \in R-P$ such that $x y \in M$. Thus $x y \in Q_{\alpha}$ for each $\alpha$ so that $x \in Q_{\alpha}$ for each $\alpha$ since $Q_{\alpha}$ is $P$-primary. Hence $x \in \bigcap_{\alpha} Q_{\alpha}=M$ and we have established that $M=M^{e c}$. Thus if $t \in P-\sqrt{ } M$, then for any positive integer $n, t^{n} \notin M$. It follows that $t^{n} \notin M^{e c}$ so that $\sqrt{ } M^{e} \subset P^{e}$. But it is easily seen that $M^{e}=\left(\bigcap_{\alpha} Q_{\alpha}\right)^{e}=\bigcap_{\alpha} Q_{\alpha}^{e}$, and $\left\{Q_{\alpha}^{e}\right\}$ is the set of $P^{e}$-primary ideals of $R_{P}$. Applying Proposition 2.4 to the ring $R_{P}$ it follows that $M^{e}$ is prime in $R_{P}$. Hence $M=M^{e c}$ is prime in $R$. Q.E.D.

2.6. Proposition. If $P$ is maximal in $R$ and if the powers of $P$ properly descend, then $\left\{P^{n}\right\}_{n=1}^{\infty}$ is the set of $P$-primary ideals and $M=\bigcap_{n=1}^{\infty} P^{n}$ is a prime ideal.

Proof. By Proposition 2.2 each $P$-primary ideal properly contained in $P^{n}$, for any positive integer $n$, is contained in $P^{n+1}$. We use this fact to show $A=\bigcap_{n=1}^{\infty} P^{n}$ is a prime ideal. If $x, y \in R-A$ we may choose integers $k, t$ such that $x \in P^{k}-P^{k+1}$ and $y \in P^{t}-P^{t+1}$, where $P^{0}=R$. Then $P^{k}=P^{k+1}+(x)$ and $P^{t}=P^{t+1}+(y)$ since there are no ideals of $R$ properly between $P^{n}$ and $P^{n+1}$ for any nonnegative $n$. Hence $P^{k+t}=\left(P^{k+1}+(x)\right)\left(P^{t+1}+(y)\right) \subseteq P^{k+t+1}+(x y)$. Since $P^{k+t} \supset P^{k+t+1}$, it follows that $x y \notin P^{k+t+1}$ so that $x y \notin A$ and $A$ is prime. 
Now if $Q$ is $P$-primary, $Q \nsubseteq A$ so $Q \nsubseteq P^{k}$ for some $k$. Therefore $Q \supseteq P^{k}$ and if $t$ is such that $Q \supseteq P^{t}$ but $Q \nsupseteq P^{t-1}$, we must have $Q=P^{t}$. This completes the proof.

As with Corollary 2.5, Corollary 2.7 follows immediately from Proposition 2.6 by passage to the quotient ring $R_{P}$. We therefore omit the proof.

2.7. Corollary. If the symbolic powers of $P$ properly descend, then $\left\{P^{(n)}\right\}_{n=1}^{\infty}$ is the set of P-primary ideals and $M=\bigcap_{n=1}^{\infty} P^{(n)}$ is prime.

3. A characterization of domains for which $\mathscr{Q}(D) \subseteq \mathscr{V}(D)$. Let $P$ be a prime ideal of $D$, a domain with identity; let $\left\{Q_{\alpha}\right\}$ be the set of $P$-primary ideals. Our purpose in this section is to establish the validity of the following statement $(*)$ :

(*): In order that each $Q_{\alpha}$ be a valuation ideal it is necessary and sufficient that $\left\{Q_{\alpha}\right\}$ be linearly ordered under $\subseteq$ and that $\bigcap_{\alpha} Q_{\alpha}$ be a prime ideal.

We first achieve a reduction to the case when $D$ is quasi-local via Theorem 3.1.

3.1. THEOREM. If $(*)$ holds when $D$ is quasi-local, then $(*)$ is true in any domain with identity.

Proof. $D_{P}$ is a quasi-local domain with maximal ideal $P D_{P}$, and $\left\{Q_{\alpha} D_{P}\right\}$ is the set of $P D_{P}$-primary ideals. Since $Q_{\alpha} D_{P} \cap D=Q_{\alpha}$, it follows that if $Q_{\alpha} D_{P}$ is a valuation ideal, then $Q_{\alpha}$ is a valuation ideal. Corollary 2.6 of [2] shows that the converse is also valid. It is clear that $\left\{Q_{\alpha} D_{P}\right\}$ is linearly ordered under $\subseteq$ if and only if $\left\{Q_{\alpha}\right\}$ is linearly ordered under $\subseteq$. Finally, the proof of Corollary 2.5 shows that if $\bigcap_{\alpha} Q_{\alpha}$ is prime then $\bigcap_{\alpha} Q_{\alpha} D_{P}$ is also prime, and the converse is obvious. All these observations establish Theorem 3.1.

We are now able to establish in Theorem 3.2 necessity of the conditions given in (*). The proof of Theorem 3.2 uses the following result:

If $R$ is a commutative ring such that the set of principal ideals of $R$ is linearly ordered under $\subseteq$, then the set of ideals of $R$ is linearly ordered under $\subseteq$.

3.2. TheOREM. Suppose $J$ is a quasi-local domain with maximal ideal $M$. If $M$ primary ideals of $J$ are valuation ideals, the set of M-primary ideals is linearly ordered under $\subseteq$ and the intersection of the set of M-primary ideals is a prime ideal.

Proof. Suppose $Q$ is $M$-primary. We first show that for $x, y \in J$,

$$
Q+(x) \subseteq Q+(y) \quad \text { or } \quad Q+(y) \subseteq Q+(x) .
$$

This is clear unless $x, y \in M$. In this case $Q^{2}+(x y)$ has radical $M$, is therefore $M$-primary, and hence is a valuation ideal. Thus $x^{2} \in Q^{2}+(x y)$ or $y^{2} \in Q^{2}+(x y)$ say $x^{2} \in Q^{2}+(x y) ; x^{2}=q+r x y$ where $q \in Q^{2}$ and $r \in J$. It follows that $x(x-r y)$ $=q \in Q^{2}$. But $Q$ is a $v$-ideal for some valuation $v$. Hence Lemma 2.8 of [2] applies to show that either $x \in Q$ or $x-r y \in Q$. Consequently $Q+(x)=Q \subseteq Q+(y)$ or $x=(x-r y)+r y \in Q+(y)$ and again $Q+(x) \subseteq Q+(y)$. It then follows that the set of principal ideals of $D / Q$ are linearly ordered under $\subseteq$ so that the ideals of $D / Q$ are linearly ordered under $\subseteq$. But this implies that the ideals of $D$ which contain 
$Q$ form a chain-this statement holds for any $M$-primary ideal $Q$. In particular, if $Q_{1}$ and $Q_{2}$ are $M$-primary, $Q_{1} \cap Q_{2}$ is also $M$-primary and therefore $Q_{1} \subseteq Q_{2}$ or $Q_{2} \subseteq Q_{1}$. We have shown that the set of $M$-primary ideals is linearly ordered under $\subseteq$. That the intersection of all $M$-primary ideals is a prime ideal is the content of Proposition 2.14 of [2].

We still wish to show that if $\left\{Q_{\alpha}\right\}$ is the set of $P$-primary ideals of a quasi-local domain $D$ with maximal ideal $P$, if $\left\{Q_{\alpha}\right\}$ is chained under $\subseteq$, and if $\bigcap_{\alpha} Q_{\alpha}=M$ is a prime ideal, then each $Q_{\alpha}$ is a valuation ideal. Lemmas 3.3 and 3.4 allow us to reduce this problem to the case when $M=(0)$.

3.3. Lemma. Let $R$ be a subring of the ring $S$, let $B$ be an ideal of $S$ and let $A_{0}=B \cap R$. Let $\phi: S \rightarrow S / B$ be the canonical homomorphism. We identify $R / A_{0}$ with $\phi(R)=(R+B) / B \simeq R /(R \cap B)$. Let $C$ be an ideal of $R$ containing $A_{0}, \bar{T}$ be a ring between $R / A_{0}$ and $S / B$ such that some ideal $\bar{N}$ of $\bar{T}$ lies over $C / A_{0}: \bar{N} \cap\left(R / A_{0}\right)$ $=C / A_{0}$. If $T=\phi^{-1}(\bar{T}), T$ is a ring between $R$ and $S$ and $N=\phi^{-1}(\bar{N})$ is an ideal of $T$ lying over $C$.

Proof. The proof is immediate and will be omitted.

3.4. Lemma. Let $A$ be an ideal of a domain $D$ and let $P_{1}$ be a prime ideal of $D$ contained in $A$ such that $A / P_{1}$ is a valuation ideal of $D / P_{1}$. Then $A$ is a valuation ideal of $D$.

Proof. Let $V$ be a valuation ring between $D$ and its quotient field such that $V$ has center $P_{1}$ on $D$. If $M$ is the maximal ideal of $V$, then $V / M=\bar{k}$ contains $D / P_{1}$ to within isomorphsm and therefore contains the quotient field $\bar{k}_{1}$ of $D / P_{1}$. By hypothesis there is a valuation ring $\bar{V}_{1}$ and an ideal $\bar{A}_{1}$ of $\bar{V}_{1}$ such that

$$
D / P_{1} \subseteq \bar{V}_{1} \subseteq \bar{k}_{1} \quad \text { and } \quad \bar{A}_{1} \cap\left(D / P_{1}\right)=A / P_{1} .
$$

The valuation ring $\bar{V}_{1}$ has an extension to a valuation ring $\bar{V}$ with quotient field $\bar{k}$. We show that $\bar{A}_{1} \bar{V} \cap \bar{V}_{1}=\bar{A}_{1}$. $\supseteq$ always holds. We consider a nonzero element $a=\sum_{i=1}^{n} a_{i} t_{i} \in \bar{A}_{1} \bar{V} \cap \bar{V}_{1}$, where $a_{i} \in \bar{A}_{1}$ and $t_{i} \in \bar{V}$. The ideal of $\bar{V}$ generated by $\left\{a_{1}, \ldots, a_{n}\right\}$ is principal and is generated by some $a_{i}$-say by $a_{1}$. Then $a=a_{1} t$ for some $t \in \bar{V}$. Hence $t=a / a_{1} \in \bar{V} \cap \bar{k}_{1}=\bar{V}_{1}$, implying that $a \in a_{1} \bar{V}_{1} \subseteq \bar{A}_{1}$. This proves that $\bar{A}_{1} \bar{V} \cap \bar{V}_{1}=\bar{A}_{1}$ so that the ideal $\bar{A}_{1} \bar{V}$ of $\bar{V}$ lies over $A / P_{1}$ in $D / P_{1}$.

We now apply Lemma 3.3 with $R=D, S=V, B=M, C=A, \bar{T}=\bar{V}$, and $\bar{N}=\bar{A}_{1} \bar{V}$. We conclude that if $\phi$ is the canonical homomorphism from $V$ onto $V / M$, then $\phi^{-1}(\bar{V})$ is a domain between $D$ and $V$ containing the ideal $\phi^{-1}\left(\bar{A}_{1} \bar{V}\right)$ lying over $A$. But $V$ and $\bar{V}$ are valuation rings so that $\phi^{-1}(\bar{V})$ is also a valuation ring [4, p. 34, Result 11.4]. Hence $A$ is a valuation ideal. Q.E.D.

Lemma 3.4 shows that in proving $(*)$ there is no loss of generality in assuming $\bigcap_{\alpha} Q_{\alpha}=(0)$, for if we show $Q_{\alpha} / M$ is a valuation ideal of the domain $D / M$, where $M=\bigcap_{\alpha} Q_{\alpha}$, then Lemma 3.4 shows that $Q_{\alpha}$ is also a valuation ideal. Also, $\left\{Q_{\alpha} / M\right\}$ is the set of $P / M$-primary ideals of $D / M$, this set is chained under $\subseteq$, and 
$\bigcap_{\alpha}\left(Q_{\alpha} / M\right)=(\overline{0})$. We therefore introduce the following notation for the remainder of this section: $J$ denotes a quasi-local domain with maximal ideal $P ;\left\{Q_{\alpha}\right\}$ is the set of $P$-primary ideals. We assume $\left\{Q_{\alpha}\right\}$ is linearly ordered under $\subseteq$ and that $\bigcap_{\alpha} Q_{\alpha}=(0)$. As in $\S 1$ we define, for $x \in J, x \neq 0, B_{x}=\bigcap_{\alpha}\left(Q_{\alpha}+(x)\right)$. Our object is to prove Theorem 3.5, from which the validity of $(*)$ will follow.

\subsection{TheOREM. Each $Q_{\alpha}$ is a valuation ideal.}

Our proof will require the following lemma.

3.6. Lemma. For $a, b, c, d \in J$ if $a / b=c / d$, then $B_{a} \subseteq B_{b}$ if and only if $B_{c} \subseteq B_{d}$.

Proof. The conclusion being symmetric, we need only prove that $B_{a} \subseteq B_{b}$ implies $B_{c} \subseteq B_{d}$. Since $B_{c}$ and $B_{d}$ are $P$-primary, $B_{c} \subseteq B_{d}$ or $B_{d} \subseteq B_{c}$. We show that if $B_{d} \subseteq B_{c}$, then $B_{d}=B_{c}$. Thus let $Q$ be a $P$-primary ideal such that $a d \notin Q$. Since $B_{a} \subseteq B_{b}$, $a \in Q+(b)$-say $a=q_{1}+r_{1} b$ where $q_{1} \in Q$ and $r_{1} \in J$. By assumption $a d \notin Q$ so that $d \notin Q$. This implies that $Q \subset B_{d}$, and hence that $Q+(d) \subseteq B_{d} \cdot Q+(d)$ is $P$ primary and contains $d$, however, so that equality holds: $B_{d}=Q+(d)$. Also, $B_{d} \subseteq B_{c} \subseteq Q+(c)$ so that $d=q_{2}+r_{2} c$ for some $q_{2} \in Q, r_{2} \in J$. Then $b c=a d=\left(q_{1}+r_{1} b\right)$ $\times\left(q_{2}+r_{2} c\right) \equiv r_{1} r_{2} b c$ modulo $Q$. Thus $\left(1-r_{1} r_{2}\right) b c \in Q$. Because $b c \notin Q$ this implies $1-r_{1} r_{2}$ is a nonunit of $J$, implying that $r_{1}$ and $r_{2}$ are units of $J$. In particular $c=r_{2}^{-1}\left(d-q_{2}\right) \in Q+(d)=B_{d}, B_{c} \subseteq B_{d}$, and our proof is complete. Q.E.D.

Proof of Theorem 3.5. We define $V=\left\{a|b| a, b \in J, b \neq 0\right.$, and $\left.B_{a} \subseteq B_{b}\right\}$. We show $V$ is a valuation ring between $J$ and its quotient field $K$ such that $Q_{\alpha} V \cap J=Q_{\alpha}$ for each $\alpha$. First, Lemma 3.6 shows that $V$ is a well-defined subset of $K$, and it is clear that $J \subseteq V$. We show $V$ is a valuation ring. If $a / b, c / d \in V$ we have $B_{a} \subseteq B_{b}$ and $B_{c} \subseteq B_{d}$. By Lemma 2.3, $B_{a c}=B_{a} B_{c} \subseteq B_{b} B_{c} \subseteq B_{b} B_{d}=B_{b d}$. Thus $a c / b d=(a / b)(c / d)$ $\in V$. Similarly, $B_{a d} \subseteq B_{b d}, B_{b c} \subseteq B_{b d}$, and hence $B_{a d-b c} \subseteq B_{a d}+B_{b c} \subseteq B_{b d}$, implying $(a d-b c) / b d=(a / b)-(c / d) \in V$. Finally, the linear ordering on the set of $P$-primary ideals implies for $a, b \in J$ that $B_{a} \subseteq B_{b}$ or $B_{b} \subseteq B_{a}$. Thus $a / b$ or $b / a$ is in $V$, and $V$ is a valuation ring.

For any $\alpha$ we have $Q_{\alpha} \subseteq Q_{\alpha} V \cap J$. And any nonzero element $x$ of $Q_{\alpha} V \cap J$ is of the form $q t$ for some $q \in Q_{\alpha}, t \in V$. We have $t=x / q$ with $x, q \in J$ so that $B_{x} \subseteq B_{q} \subseteq Q_{\alpha}$. Hence $x \in Q_{\alpha}$ and $Q_{\alpha} V \cap J=Q_{\alpha}$ as we wished to show.

We remark that we have actually established the validity of the following Corollary 3.7. The question of the status of this result was raised in [2].

3.7. Corollary. Suppose $P$ is prime in the domain $D$ and that each $P$-primary ideal is a valuation ideal. Then there exists a valuation $v$, nonnegative on $D$, such that each P-primary ideal is a v-ideal.

4. Further properties of domains for which $\mathscr{Q}(D) \subseteq \mathscr{V}(D)$. We have shown in $\S 3$ that if $\left\{Q_{\alpha}\right\}$, the set of $P$-primary ideals of a domain $D$, is chained under $\subseteq$ and if $\bigcap_{\alpha} Q_{\alpha}$ is a prime ideal of $D$, then each $Q_{\alpha}$ is a valuation ideal. In fact, there is a single valuation $v$ such that each $Q_{\alpha}$ is a $v$-ideal. We investigate in this section the 
uniqueness of such a valuation $v$, the dependence of the condition " $\bigcap_{\alpha} Q_{\alpha}$ is prime" on the condition " $\left\{Q_{\alpha}\right\}$ is chained under $\subseteq$ ", and some questions raised in [1] and [2] concerning the structure of the set of prime ideals of a domain in which primary ideals are valuation ideals.

Before proving Theorem 4.1 we need some additional terminology. Following $[1, \mathrm{p}$. 252] we say that a prime ideal $P$ of a commutative ring $R$ is branched provided there exists $Q \neq P$ such that $Q$ is $P$-primary; otherwise we say $P$ is unbranched. If $R$ is a Prüfer domain-in particular if $R$ is a valuation ring-then $P$ is branched if and only if $P$ properly contains the union of the chain of all prime ideals properly contained in $P$ [1, p. 252, Lemma 3.4]. If $D$ is a domain with quotient field $K$, if $V$ is a valuation ring between $D$ and $K$ having maximal ideal $M$, and if $P$ is the center of $V$ on $D$, we say $V$ has absolute center $P$ on $D$ provided $M$ is the only prime ideal of $V$ lying over $P$.

4.1. Theorem. Let $P$ be a branched prime ideal of a domain $D$ with quotient field $K$ such that the set $\left\{Q_{\alpha}\right\}$ of P-primary ideals is linearly ordered under $\subseteq$. Then these statements are equivalent:

(a) $\bigcap_{\alpha} Q_{\alpha}$ is a prime ideal.

(b) Each $Q_{\alpha}$ is a valuation ideal.

(c) Some $Q_{\alpha} \neq P$ is a valuation ideal.

(d) There exists $Q_{\alpha} \neq P$ and a valuation ring $V$ between $D$ and $K$ such that $Q_{\alpha} V \neq P V$.

(e) $P$ is the absolute center of some valuation ring with branched maximal ideal.

Proof. (a) $\leftrightarrow$ (b): By $\S 3$.

(b) $\rightarrow$ (c): Obvious.

(c) $\rightarrow$ (d): Clear.

(d) $\rightarrow$ (e): Let $Q_{\alpha}$ and $V$ be as in (d). Then we have $Q_{\alpha} V \subset P V \subseteq M=(P V)^{1 / 2}$.

Since $V$ is a valuation ring, $M$ is a prime ideal; also $M=\left(Q_{\alpha} V\right)^{1 / 2}$ where $Q_{\alpha} V \subset M$. It follows that $M$ is branched [1, p. 248, Lemma 1.6]. Therefore $W=V_{M}$ is a valuation ring having absolute center $P$ on $D$ and such that the maximal ideal $M=M V_{M}$ of $W$ is branched.

(e) $\rightarrow$ (a): Let $V$ be a valuation ring between $D$ and $K$ having absolute center $P$ on $D$ and the maximal ideal $M$ of $V$ is branched. Then if $\left\{A_{\beta}\right\}$ is the set of $M$ primary ideals, $M^{\prime}=\bigcap_{\beta} A_{\beta}$ is a prime ideal of $V$ properly contained in $M$ [2, p. 241, Proposition 2.14]. Because $V$ has absolute center $P$ on $D$, it follows that $M^{\prime} \cap D=\left(\bigcap_{\beta} A_{\beta}\right) \cap D=\bigcap_{\beta}\left(A_{\beta} \cap D\right)=P^{\prime}$ is a prime ideal of $D$ properly contained in $P$. Hence $\left\{A_{\beta} \cap D\right\}$ is a collection of $P$-primary ideals whose intersection does not have radical $P$. By Lemma $2.1, \bigcap_{\beta}\left(A_{\beta} \cap D\right)=\bigcap_{\alpha} Q_{\alpha}$ and (a) holds. Q.E.D.

4.2. COROLlary. If the ascending chain condition for prime ideals holds in the domain $D$ and if P-primary ideals of $D$ are linearly ordered for each prime ideal $P$ of $D$, then $D$ is a Prüfer domain. 
Proof. Let $M$ be a proper prime ideal of $D$. Since the a.c.c. for prime ideals holds in $D$, there is a prime ideal $M_{1} \subset M$ such that there are no prime ideals of $D$ properly between $M_{1}$ and $M$. By Lemma 3.2 of [2], $M_{1}$ is an intersection of a collection of $M$-primary ideals. By Lemma 2.1, $M_{1}$ is therefore the intersection of all $M$-primary ideals. By Theorem 4.1, primary ideals of $D$ are valuation ideals. Theorem 3.8 of [2] then shows $D$ is Prüfer.

In a preprint of this paper we left as an open question whether the hypothesis that $\left\{Q_{\alpha}\right\}$ is linearly ordered in Theorem 4.1 implies that $\bigcap_{\alpha} Q_{\alpha}$ is prime. The following example, communicated to us by M. Nagata and T. Kikuchi, shows that the answer to this question is negative. Let $V$ be a valuation ring with unbranched maximal ideal $M$ and quotient field $K$ such that for some element $t$ of $M, t$ has no square root in $K$. (See [2, p. 248] for such a construction.) If $s$ is a square root of $t$ in an extension field of $K$, we let $D=V[s] . D$ is integral over $V$; let $P$ be a prime ideal of $D$ lying over $M$. Since $s^{2}$ is in $P, s$ is in $P$ so that $P=M D+(s)$. If $Q$ is $P$-primary, $Q \cap V$ is $M$-primary, and hence $Q \cap V=M$. It follows that $M D \subseteq Q \subseteq M D+(s)$ so that $Q=M D$ or $Q=P$. Therefore, the set of $P$-primary ideals is linearly ordered while $M D$, the intersection of the set of $P$-primary ideals, is not prime in $D$.

4.3. THEOREM. Suppose $P$ is a branched prime ideal of a domain $D$ such that $P$-primary ideals are valuation ideals. If $\left\{Q_{\alpha}\right\}$ is the set of $P$-primary ideals and if some $Q_{\beta} \neq P$ is such that $Q_{\beta} W \cap D=Q_{\beta}$, where $W$ is a valuation ring between $D$ and its quotient field $K$, then $Q_{\alpha} W \cap D=Q_{\alpha}$ for each $\alpha$.

Proof. Since $Q_{\beta} \neq P, Q_{\beta} W \subset P W \subseteq M=\sqrt{ }(P W)=\sqrt{ }\left(Q_{\beta} W\right)$ so that $M$ is a branched prime ideal of $W$. If $M^{\prime}$ is the intersection of the set of $M$-primary ideals of $W, M^{\prime}$ is a prime ideal properly contained in $M$. By choice of $M, P=M \cap D$ $\supset M^{\prime} \cap D=P^{\prime}, P^{\prime}$ is prime in $D$ and is the intersection of a collection of $P$-primary ideals. By Lemma 2.1, $P^{\prime}$ is the intersection of the collection of all $P$-primary ideals. Thus $W_{M}$ is a valuation ring containing $W$ having absolute center $P$ on $D$ and there exist $P$-primary ideals distinct from $P$ which are contractions of ideals of $W_{M}$. Finally, if we show that each $Q_{\alpha}$ is the contraction of an ideal of $W_{M}$, it is clear that each $Q_{\alpha}$ is also the contraction of an ideal of $W$. We therefore assume without loss of generality that $W$ has absolute center $P$ on $D$.

We let $w$ be a valuation associated with $W$. Then $P D_{P}$-primary ideals of $D_{P}$ are valuation ideals, $Q_{\beta} D_{P} \neq P D_{P}$ is $P D_{P}$-primary and is a $w$-ideal, and if each $Q_{\alpha} D_{P}$ is a $w$-ideal, each $Q_{\alpha}$ is also a $w$-ideal [2, pp. 239-240]. Hence we may further assume that $D$ is quasi-local and $P$ is maximal in $D$.

Now under the assumptions that $D$ is quasi-local with maximal ideal $P$ and that $W$ has absolute center $P$ on $D$, we prove Theorem 4.3. We denote by $\left\{C_{\beta}\right\}$ the set of $P$-primary ideals which are $w$-ideals. As previously observed, $\bigcap_{\beta} C_{\beta}=P^{\prime}=\bigcap_{\alpha} Q_{\alpha}$. We wish to show $\left\{C_{\beta}\right\}=\left\{Q_{\alpha}\right\}$-that is, we wish to prove that if $Q$ is $P$-primary, then $Q W \cap D \subseteq Q$. Since $Q \nsubseteq \bigcap_{\beta} C_{\beta}$, the linear ordering of the set $\left\{Q_{\alpha}\right\}$ implies $C_{\beta} \subset Q$ 
for some $\beta$. We choose $x=q \xi \in Q W \cap D$ where $q \in Q, \xi \in W$. We show $x \in Q$. The ideals $C_{\beta}+(x)$ and $C_{\beta}+(q)$ are $P$-primary. Hence either $C_{\beta}+(x) \subseteq C_{\beta}+(q) \subseteq Q$ and $x \in Q$, or $C_{\beta}+(q) \subseteq C_{\beta}+(x)$. If $q \in C_{\beta}$ we have $x \in C_{\beta} W \cap D=C_{\beta} \subseteq Q$. And if $q \notin C_{\beta}$, then $q=r+s x=r+s q \xi$ for some $r \in C_{\beta}, s \in D$. Thus $q(1-s \xi)=r \in C_{\beta}$. Because $q \notin C_{\beta}$ and $C_{\beta}$ is a $w$-ideal, it follows that $w(1-s \xi)>0$. Therefore we must have $w(s \xi)=w(s)+w(\xi)=w(1)=0$. But $w(s)$ and $w(\xi)$ are nonnegative; consequently $w(s)=w(\xi)=0$. Therefore $s$ is a unit of $W$, and hence is a unit of $D$. Thus $x=$ $s^{-1}(q-r) \in C_{\beta}+(q) \subseteq Q$. Q.E.D.

We can prove the following result, which is related to Theorem 4.3. All the ideas of the proof are included in previous results, so we shall not supply a proof.

If $P$-primary ideals of the domain $D$ are valuation ideals and if $P_{1}$ is a prime ideal of $D$ contained in each $P$-primary ideal $Q_{\alpha}$, then given any valuation ring $V$ centered on $P_{1}, V$ contains a valuation ring $V_{1}$ containing $D$ such that each $P$-primary ideal is the contraction of an ideal of $V_{1}$.

If $P$ is a branched prime ideal of a domain $D$ such that $P$-primary ideals are valuation ideals, the proof of Theorem 4.3 establishes the existence of a valuation ring $V$ between $D$ and its quotient field such that $V$ has center $P$ on $D$ and such that each $P$-primary ideal is the contraction of an ideal of $V$. In case $P$ is maximal in $D$ and the intersection of the set of $P$-primary ideals is zero, we obtain the uniqueness of $V$ in Theorem 4.4.

4.4. Theorem. Suppose $P$ is a maximal ideal of the domain D such that P-primary ideals are valuation ideals and such that the intersection of all P-primary ideals is (0). If $\left\{Q_{\alpha}\right\}$ is the set of $P$-primary ideals, there is a unique valuation ring $V$ between $D$ and its quotient field $K$ such that $Q_{\beta} V \cap D=Q_{\beta}$ for some $Q_{\beta} \neq P$. Moreover, this valuation ring has rank one.

Proof. Consider some $Q_{\gamma} \neq P$. As noted previously, there is a valuation ring $W$ with absolute center $P$ on $D$ such that $Q_{\gamma} W \cap D=Q_{\gamma} \cdot\left(Q_{\gamma} W\right)^{1 / 2}=(P W)^{1 / 2}=M$ is necessarily the maximal ideal of $W$ and $M$ is branched. If $M^{\prime}$ is the intersection of the set of $M$-primary ideals, then $M^{\prime} \cap D$ is the intersection of all $P$-primary ideals-that is, $M^{\prime} \cap D=(0)$. Because $W$ is an overring of $D$, this implies $M^{\prime}=(0)$ -that is, $W$ has rank one.

Now suppose $V_{1}$ is any valuation ring between $D$ and $K$ such that $Q_{\beta} V_{1} \cap V=Q_{\beta}$ for some $Q_{\beta} \neq P$. By Theorem 4.3, $Q_{\alpha} V_{1} \cap V=Q_{\alpha}$ for each $\alpha$. For $x \in D$ we define $C_{x}=x V_{1} \cap D$. If $x \in P-\{0\}$, there exists $Q_{\alpha}$ such that $x \notin Q_{\alpha}=Q_{\alpha} V_{1} \cap D$. Therefore $x \notin Q_{\alpha} V_{1}, Q_{\alpha} V_{1} \subseteq x V_{1}$, and $Q_{\alpha} \subseteq C_{x}$. This shows that $C_{x}$ is $P$-primary and consequently $B_{x} \subseteq C_{x}$ where, as in $\S 2, B_{y}$ is defined for $y$ in $D$ as $\bigcap_{\alpha}\left(Q_{\alpha}+(y)\right)$. However $x V_{1} \subseteq B_{x} V_{1}$ implies $C_{x} \subseteq B_{x} V_{1} \cap D=B_{x}$. Therefore $B_{x}=C_{x}$ for any $x \in D$.

We consider now any nonzero element $a / b$ of $K$. If $v_{1}$ is a valuation associated with $V_{1}, v_{1}(a / b) \geqq 0$ if and only if $a V_{1} \subseteq b V_{1}$. But since $C_{a}$ and $C_{b}$ are $v_{1}$-ideals, $a V_{1} \subseteq b V_{1}$ if and only if $C_{a} \subseteq C_{b}$-that is, if and only if $B_{a} \subseteq B_{b}$. This shows that $V_{1}$ is uniquely determined by $D$. Hence $V_{1}=W$ and $V_{1}$ has rank one. 
Example 5.2 of $\S 5$ shows that the assumption $\bigcap_{\alpha} Q_{\alpha}=(0)$ in Theorem 4.4 cannot be dropped.

4.5. Proposition. Let $D$ and $P$ be as in Theorem 4.4. The rank one valuation ring $V$ whose existence is established in Theorem 4.4 is discrete if and only if $P \supset P^{2}$.

Proof. We suppose $P \supset P^{2}$. Then for $n>1, P^{n}$ is a valuation ideal which is not prime. By Corollary 1.4 of [1], $P^{n}$ is not idempotent. In particular, $P^{n} \supset P^{n+1}$. We conclude that the powers of $P$ properly descend. By Proposition $2.6\left\{P^{n}\right\}_{n=1}^{\infty}$ is the set of $P$-primary ideals of $D$. Thus if $p \in P-P^{2}$ and if $a / b$ is a nonzero nonunit of $V$-that is, if $B_{a}=P^{r} \subset B_{b}=P^{s}, B_{a} \subseteq P^{s+1}=B_{b} B_{p}=B_{b p}$ so that $a / b p \in V$. Therefore $a / b \in p V, p V$ is the maximal ideal of $V$, and hence $V$ is discrete.

Conversely, if $V$ is discrete with maximal ideal $M$, then $\bigcap_{n=1}^{\infty}\left(M^{n} \cap D\right)=(0)$ so that $M^{n} \cap D \subset P$ for some n. But $P^{n} \subseteq M^{n} \cap D$, and therefore $P^{n} \subset P$. In particular, $P \supset P^{2}$.

Example 5.8 will show that even in the case when $D$ is quasi-local with maximal ideal $M$, primary ideals of $D$ are valuation ideals, $M \supset M^{2}$, and $\bigcap_{n=1}^{\infty} M^{n}=(0)$, $D$ need not be a valuation ring.

5. S-domains. We conclude by considering some questions raised in [1], where it was shown that in a weak $S$-domain, and hence in an $S$-domain, primary ideals are valuation ideals. The questions of whether a domain in which primary ideals are valuation ideals need be an $S$-domain or a weak $S$-domain were left open in [1]. We show in Example 5.8 that $\mathscr{Q}(D) \subseteq \mathscr{V}(D)$ does not imply $D$ is a weak $S$-domain. Example 5.9 shows that a weak $S$-domain need not be an $S$-domain. And Example 5.2 shows that no natural generalization of Theorem 4.4 is valid. Section 5 ends with some questions concerning domains in which primary ideals are valuation ideals which we are unable to answer.

5.1. Proposition. Let $D$ be a domain with quotient field $F$ such that primary ideals of $D$ are valuation ideals, let $K$ be a field containing $F$, and let $W$ be a valuation ring with unbranched maximal ideal $M$ such that $W=K+M$. If $D_{1}=D+M, D_{1}$ has the following properties:

(a) $2\left(D_{1}\right) \subseteq \mathscr{V}\left(D_{1}\right)$

(b) Each ideal of $D_{1}$ containing $M$ is of the form $A+M$ for some ideal $A$ of $D$. Further, $D_{1} /(A+M) \simeq D / A$ so $A$ is maximal, prime, or primary, respectively, in $D$ if and only if $A+M$ is maximal, prime, or primary, respectively, in $D_{1}$.

(c) If $Q$ is a primary ideal of $D$ and if $V$ is any valuation ring between $D$ and $F$ such that $Q V \cap D=Q$, then for any extension $V_{\alpha}$ of $V$ to $K, V_{\alpha}+M$ is a valuation ring and $(Q+M)\left(V_{\alpha}+M\right) \cap D_{1}=Q+M$.

Proof. We first prove (b). To show any ideal of $D_{1}$ compares with $M$ under $\subseteq$ it suffices to prove that this is true for any principal ideal $x D_{1}$. For $x \in M, x D_{1} \subseteq M$. If $x \in D_{1}-M, x$ is a unit of $W$. Hence $m \in M$ implies $m / x \in M$ also. Therefore 
$m \in M x \subseteq D_{1} x$. We obtain the other assertions of (b) by considering the canonical homomorphism of $D_{1}$ onto $D_{1} / M \simeq D$.

To prove (c) we apply Lemma 3.3. Under the natural homomorphism $\phi$ from $W$ onto $W / M \simeq K$, the inverse image of any valuation ring $V_{\alpha}$ with quotient field $K$ is again a valuation ring, and this inverse image is $V_{\alpha}+M$. $\phi$ sends $D_{1}$ onto $D_{1} / M \simeq D$ and sends $V+M$ onto $V$. Since $V_{\alpha}$ is an extension of $V$, each ideal of $V$ is the contraction of an ideal of $V_{\alpha}$. In particular $Q V$ is the contraction of an ideal of $V_{\alpha}$ so that $Q$ is also the contraction of an ideal of $V_{\alpha}$. Lemma 3.3 then shows that $\phi^{-1}(Q)=Q+M$ is the contraction of an ideal of $\phi^{-1}\left(V_{\alpha}\right)=V_{\alpha}+M$. Therefore $Q+M$ must be the contraction to $D+M$ of $(Q+M)\left(V_{\alpha}+M\right)$.

To prove (a) we let $Q$ be a $P$-primary ideal of $D_{1},(0) \subset P \subset D_{1}$. Since primary ideals of $D$ are valuation ideals, (c) and (b) show that if $P \supset M, Q$ is a valuation ideal. If $P \subset M$ we choose $a \in M-P$. If $s \in K$, then $s a \in M$ so that $s=s a / a \in\left(D_{1}\right)_{P}$. Hence $K+M=W$, a valuation ring, is contained in $\left(D_{1}\right)_{P}$. Thus $\left(D_{1}\right)_{P}$ is a valuation ring so that $Q$ is a valuation ideal. Finally, we consider the case $P=M$. In this case $Q M$ is an ideal of $W$ with radical $M$. Because $M$ is unbranched, $M=Q M \subseteq Q$. Hence $Q$ is a valuation ideal and our proof is complete.

5.2. ExAmple. In the notation of Proposition 5.1 we let $K=F_{0}(X, Y)$ where $X$ and $Y$ are indeterminates over the field $F_{0}$ and we let $D=\left(F_{0}[X]\right)_{(X)}$. On the field $K\left(\left\{X_{n}\right\}_{n=1}^{\infty}\right),\left\{X_{n}\right\}$ a set of indeterminates over $K$, there is a valuation ring $W$ with unbranched maximal ideal $M$ such that $W=K+M[2$, p. 248]. We then define $D_{1}=D+M . D$ is a rank one discrete valuation ring which has infinitely many extensions to the field $K$. If $N=X D$ is the maximal ideal of $D, 5.1$ (b) shows $D_{1}$ is quasi-local with maximal $N+M . N+M$ is branched and 5.1 (c) shows that there are infinitely many valuation rings $V_{\alpha}$ between $D_{1}$ and its quotient field such that each $(N+M)$-primary ideal of $D_{1}$ is the contraction of an ideal of $V_{\alpha}$ for each $\alpha$. This example shows that in Theorem 4.4 the assumption that $\bigcap_{\alpha} Q_{\alpha}=(0)$ is essential.

Before presenting the examples related to $S$-domains we require several preliminary results.

5.3. Lemma. For $1 \leqq i \leqq n$ let $V_{i}$ be a valuation ring with maximal ideal $M_{i}$ such that $V_{i}$ has quotient field $K$. If $V_{i} \ddagger V_{j}$ for $i \neq j$, then for any $i, 1 \leqq i \leqq n, \bigcap_{j \neq i} M_{j} \ddagger V_{i}$.

Proof. We let $v_{i}$ be a valuation associated with the valuation ring $V_{i}, 1 \leqq i \leqq n$. The domain $V=V_{1} \cap \cdots \cap V_{n}$ is a semi-quasi-local Prüfer domain with exactly $n$ maximal ideals $N_{1}=M_{1} \cap V, \ldots, N_{n}=M_{n} \cap V$; further, $V_{i}=V_{N_{i}}$ for each $i$ [5, p. 54], [3, pp. 182-184]. Therefore we may choose $x \in\left(\bigcap_{j \neq i} N_{j}\right)-N_{i}$ and $y \in N_{i}$ - $\left(\bigcup_{j \neq i} N_{j}\right)$. Hence $v_{i}(y)>0, v_{i}(x)=0, v_{j}(y)=0$, and $v_{j}(x)>0$ for each $j \neq i$. Consequently, $x / y \in\left(\bigcap_{j \neq i} M_{j}\right)-V_{i}$.

5.4. Lemma. Let $A$ be an ideal of a domain $D$ and let $P$ be a prime ideal of $D$ not containing $A$. Then $A_{A \cap P}=D_{P}$. 
Proof. That $A_{A \cap P} \subseteq D_{P}$ is clear. If $x=d / n \in D_{P}$ where $d \in D$ and $n \in D-P$, then for $b \in A-P, b d \in A$ and $b n \in A-P$. Hence $x=b d / b n \in A_{A \cap P}$. Q.E.D.

5.5. COROLLARY. Let $\left\{V_{i}\right\}_{i=1}^{n}$ be a finite family of valuation rings having a common quotient field $K$ such that $V_{i} \ddagger V_{j}$ for any $i \neq j$. Let $M_{i}$ be the maximal ideal of $V_{i}$, let $V=\bigcap_{i=1}^{n} V_{i}$, and let $P_{1}$ be a prime ideal of $V_{1}$ properly contained in $M_{1}$. If $M=\bigcap_{i=1}^{n} M_{i}$ and if $P=P_{1} \cap M_{2} \cap \cdots \cap M_{n}$, then $\left(V_{1}\right)_{P_{1}}=M_{M-P}$.

Proof. Because $V$ is a Prüfer domain, $\left(V_{1}\right)_{P_{1}}=V_{P_{1} \cap V} \supset V_{1}=V_{M_{1} \cap V}$; therefore $P_{1} \cap V \subset M_{1} \cap V$. Thus $P_{1} \cap V$ is a nonmaximal prime ideal of $V$, so that $P_{1} \cap V$ $\neq \bigcap_{i=1}^{n}\left(M_{i} \cap V\right)=\bigcap_{i=1}^{n} M_{i}=M$. Therefore $P_{1} \cap V$ is a prime ideal of $V$ not containing $M$. By Lemma 5.4, $V_{P_{1} \cap V}=\left(V_{1}\right)_{P_{1}}=M_{M \cap P_{1} \cap V}=M_{P} \equiv M_{M-P}$.

5.6. COROLlaRY. Let $\left\{V_{i}\right\}_{i=1}^{n}$ be a finite family of valuation rings having a common quotient field $K$ such that $V_{i} \ddagger V_{j}$ for $i \neq j$ and such that each $V_{i}$ contains some fixed field $F ; M_{i}$ the maximal ideal of $V_{i}$. Let $D=F+M$ where $M=M_{1} \cap \cdots \cap M_{n}$. $D$ is a quasi-local domain with maximal ideal $M$ and is not a valuation ring if $n>1$ or if $V_{1} \neq F+M_{1}$. If $P$ is a nonmaximal prime ideal of $D, P=P_{\alpha} \cap D$ for some nonmaximal prime $P_{\alpha}$ of some $V_{i}$, and $D_{P}=\left(V_{i}\right)_{P_{\alpha}}$ is a valuation ring.

Proof. Let $x \in D-M ; x=a+m$ where $a \in F-\{0\}, m \in M$. Then $1 / x-1 / a$ $=-m / a(a+m)$. Since $a(a+m)$ is a unit of each $V_{i}, m / a(a+m) \in M$. Hence $1 / x \in F$ $+M=D$. Thus $M$ is the unique maximal ideal of $D$. If $m \in M_{1}-M_{2}$, then neither $m$ nor $1 / m$ is in $D$ so $D$ is not a valuation ring.

If $P$ is a nonmaximal prime of $D, P$ is the center of some valuation ring $V$ between $D$ and $K$. Since $V \supseteq M_{1} \cap \cdots \cap M_{n}=M, V \supseteq V_{i}$ for some $i$ or $V \subseteq V_{i}$ for some $i$. The maximal ideal of $V_{i}$ contracts to $M$ on $D$ and is therefore not contained in the maximal ideal of $V$. Therefore $V_{i} \subset V$ so that $V=\left(V_{i}\right)_{P_{\alpha}}$ for some prime $P_{\alpha}$ of $V_{i}$. Further, $P_{\alpha}$ is the maximal ideal of $V$ and $P_{\alpha} \cap D=P \subset M_{i} \cap D=M$. It follows that $P_{\alpha} \subset M_{i}$. By Corollary 5.5, $\left(V_{i}\right)_{P_{\alpha}}=M_{P_{\alpha} \cap M}$. But clearly $M_{P_{\alpha} \cap M} \subseteq D_{P_{\alpha} \cap D}$ $=D_{P}$. The reverse containment is clear. Q.E.D.

5.7. Proposition. Let $V_{1}$ and $V_{2}$ be distinct valuation rings with a common quotient field $K$ such that $V_{2}=F+M_{2}$ for some field $F$ contained in $V_{1}$ and $V_{2}$, where $M_{i}$ is the maximal ideal of $V_{i}$. If $M_{1}$ is unbranched, then primary ideals of the domain $D=F+\left(M_{1} \cap M_{2}\right)$ are valuation ideals.

Proof. By Corollary 5.6, $D$ is quasi-local with maximal ideal $M=M_{1} \cap M_{2}$. Further if $P$ is a nonmaximal prime of $D, D_{P}$ is a valuation ring. Hence if $Q$ is a primary ideal of $D$ such that $\sqrt{ } Q \neq M, Q$ is a valuation ideal.

Therefore we show that if $Q$ is $M$-primary, $Q$ is a valuation ideal. Let $v_{i}$ be a valuation associated with the valuation ring $V_{i}$. We show that $Q$ is a $v_{2}$-ideal. To do this we need to show that if $x \in D$ and if $v_{2}(x) \geqq v_{2}(q)$ for some $q \in Q$, then $x \in Q$ [7, p. 340]. We first consider the case $v_{2}(x)>v_{2}(q)$. Then if $v_{1}(x)>v_{1}(q), x / q \in M$ and $x \in q M \subseteq Q$. If $v_{1}(x) \leqq v_{1}(q)$, we first observe that $v_{1}(x)>0$. This is true since 
$x \in D$ implies $x=y+m$ for some $y \in F, m \in M_{1} \cap M_{2}$. Because $v_{2}(x) \geqq v_{2}(q)>0$ it follows that $y=0$. Hence $x \in M_{1} \cap M_{2}$ and $v_{1}(x)>0$. Because $M_{1}$ is unbranched, $\left(x V_{1}\right)^{1 / 2}=P_{1} \subset M_{1}$. By Corollary 5.5, $\left(V_{1}\right)_{P_{1}}=M_{M-\left(P_{1} \cap M\right)}$. In particular, $P_{1} \cap M$ $=P_{1} \cap M_{2} \subset M$. We choose $s \in M-P_{1}$. It follows that $v_{1}\left(s^{k}\right)<v_{1}(x)$ for each positive integer $k$. Because $Q$ has radical $M, s^{n} \in Q$ for some positive integer $n$. The elements $q+s^{n}$ and $q+s^{n+1}$ are in $Q$ and one of these has $v_{2}$-value $\leqq v_{2}(q)$. If $v_{2}\left(s^{n}\right) \neq v_{2}(q), q+s^{n}$ has this property. And if $v_{2}\left(s^{n}\right)=v_{2}(q), v_{2}\left(s^{n+1}\right) \neq v_{2}(q)$ so $q+s^{n+1}$ has the desired property. Thus there is an integer $k$ such that $u=q+s^{k} \in Q$ and $v_{2}(u) \leqq v_{2}(q)$. It follows that $v_{1}(u)=v_{1}\left(s^{k}\right)<v_{1}(x)$ and $v_{2}(u) \leqq v_{2}(q)<v_{2}(x)$. As previously shown, this implies that $x \in u M \subseteq Q$.

On the other hand, if $v_{2}(x)=v_{2}(q)$, then $v_{2}(x / q)=0$ and $x / q=r+m$ for some nonzero $r$ in $F, m \in M_{2}$. Then $x-r q=q m$. Since $m \in M_{2}, v_{2}(q m)>v_{2}(q)$. But $x-r q \in M_{1}$ since $x, q \in M_{1}$. Thus $q m \in D, v_{2}(q m)>v_{2}(q)$. We have already established that $q m$ then belongs to $Q$. As $q \in Q$ and $r \in F \subseteq D$, it follows that $x=r q$ $+q m \in Q$. This completes the proof.

We remark that Proposition 5.7 will generalize to the case of $n$ valuation rings $V_{1}, V_{2}, \ldots, V_{n}$ each containing a fixed field $F$ such that at least $n-1$ of the $M_{i}$ 's are unbranched, and such that there are no containment relations among the $V_{i}$ 's.

5.8. Example. We denote by $A$ the field of algebraic numbers and by $A((X))$ the quotient field of the domain of formal power series in one indeterminate $X$ over $A$. The field $A((X))$ is uncountable while $A$ is countable so that $A((X))$ has infinite transcendence degree over $A$. We denote by $B$ a transcendence basis of $A((X))$ over $A$ such that $1 / X \in B$. Using the same construction that is outlined in [2, p. 248] we obtain a valuation ring $V$ with maximal ideal $M$ such that $A(B)$ is the quotient field of $V, B \subseteq M$, and $V=A+M$. $V$ has an extension to a valuation ring $V_{1}$ with quotient field $A((X))$. If $M_{1}$ is the maximal ideal of $V_{1}, A \simeq V / M$ is isomorphic to a subfield of $V_{1} / M_{1}$. Because $A((X))$ is algebraic over $A(B), V_{1} / M_{1}$ is algebraic over $A$. Since $A$ is algebraically closed we must have $A=V_{1} / M_{1}$. However, $A \subseteq V_{1}$ and $A \cap M=(0)$. Consequently, $V_{1}=A+M_{1}$. Finally, $A((X))$ algebraic over $A(B)$ implies that the ordinal type of the set of prime ideals of $V$ is the same as the ordinal type of the set of prime ideals of $V_{1}$. Hence the maximal ideal of $V_{1}$ is also unbranched. Finally, for $V_{2}$ we take the rank one discrete valuation ring $V_{2}=A[[X]]=A+M_{2}$ where $M_{2}=X V_{2}$ is the maximal ideal of $V_{2}$.

By Proposition 5.7, primary ideals of the domain $D=A+\left(M_{1} \cap M_{2}\right)$ are valuation ideals. Also, $D$ is quasi-local with maximal ideal $M=M_{1} \cap M_{2}$ and $M \neq M^{2}$. By Proposition 2.6, $\left\{M^{n}\right\}_{n=1}^{\infty}$ is the set of $M$-primary ideals. Since $M \subseteq M_{2}$, $\bigcap_{n=1}^{\infty} M^{n}=(0)$ in this case. Hence $D$ is not a weak $S$-domain. There is, in fact, an uncountable chain of prime ideals properly between $M$ and (0).

5.9. EXAMPLE. Let $\left\{X_{i}\right\}_{i=1}^{\infty}$ be a countable collection of indeterminates over a field $K$ and for each $i$ we set $Y_{i}=1 / X_{i}$. We define valuations $v_{1}$ and $v_{2}$ on $K\left(\left\{X_{i}\right\}_{1}^{\infty}\right)$ by defining them on $K\left[\left\{X_{i}\right\}\right]$ and $K\left[\left\{Y_{i}\right\}\right]$ as follows, then taking the canonical extension in each case to $K(\{X\}) . v_{1}$ is defined on $K\left[\left\{X_{i}\right\}\right]$ and has value group $G_{1}$, 
the countable weak direct sum of the additive group $Z$ of integers, ordered lexicographically; $v_{2}$ is defined on $K\left[\left\{Y_{i}\right\}\right]$ and has value group $G_{2}$, the countable weak direct sum of $Z$ with the reverse lexicographic ordering. $v_{1}$ is defined on a nonzero monomial $a X_{1}^{e_{1}} \ldots X_{n}^{e_{n}}$ to be $\left(e_{1}, \ldots, e_{n}, 0, \ldots\right)$, and $v_{1}(f)$, for a nonzero element $f$ of $K\left[\left\{X_{i}\right\}\right]$ as the minimum of the $v_{1}$-values of the nonzero monomials in $f . v_{2}$ is similarly defined on $K\left[\left\{Y_{i}\right\}\right]$ with the role of the $X_{i}$ 's played by the $Y_{i}$ 's. If $V_{i}$ is the valuation ring of $v_{i}, V_{i}=K+M_{i}$ where $M_{i}$ is the maximal ideal of $V_{i}$; also, $V_{1}$ and $V_{2}$ are independent and $M_{1}$ is unbranched. Hence primary ideals of

$$
D=K+\left(M_{1} \cap M_{2}\right)
$$

are valuation ideals. The ideal $M_{2}$ is branched. If $P_{2}$ is the intersection of the set of $M_{2}$-primary ideals, then $M_{1} \cap P_{2}$ is the intersection of all $\left(M_{1} \cap M_{2}\right)$-primary ideals of $D$ and there are no prime ideals of $D$ properly between $M_{1} \cap M_{2}$ and $M_{1} \cap P_{2}$. Yet there is a countable chain of prime ideals of $D$ distinct from $M_{1} \cap M_{2}$ which are not contained in $M_{1} \cap P_{2}$. It follows that $D$ is a weak $S$-domain but not a strong $S$-domain.

We conclude by listing some questions concerning primary ideals and valuation ideals which we are unable to answer. Where they are meaningful we give the local form of the question. We have not been able to settle these questions in the local or global case. $P$ denotes a branched prime ideal of a domain $D$.

1. Suppose each $P$-primary ideal is a valuation ideal, that $Q \neq P$ is $P$-primary, and that $P_{1}$ is a prime ideal contained in $Q$.

(a) Are $P / P_{1}$-primary ideals of $D / P_{1}$ valuation ideals?

(b) Is $P_{1}$ contained in each $P$-primary ideal?

2. Suppose $\mathscr{Q}(D) \subseteq \mathscr{V}(D)$ and $D_{1}$ is the integral closure of $D$. Are primary ideals of $D_{1}$ valuation ideals?

We remark that questions 1 (a) and 1(b) are equivalent. If $D_{1}$ is an arbitrary overring of $D$ integral over $D, \mathscr{Q}(D) \subseteq \mathscr{V}(D)$ does not imply $\mathscr{2}\left(D_{1}\right) \subseteq \mathscr{V}\left(D_{1}\right)$.

\section{REFERENCES}

1. R. Gilmer, $A$ class of domains in which primary ideals are valuation ideals, Math. Ann. 161 (1965), 247-254.

2. R. Gilmer and J. Ohm, Primary ideals and valuation ideals, Trans. Amer. Math. Soc. 117 (1965), 237-250.

3. W. Krull, Allgemeine Bewertungstheorie, J. Reine Angew. Math. 167 (1932), 160-196.

4. M. Nagata, Local rings, Interscience, New York, 1962.

5. —_, On the theory of Henselian rings, Nagoya Math. J. 5 (1953), 45-57.

6. O. Zariski and P. Samuel, Commutative algebra, Vol. I, Van Nostrand, Princeton, N. J., 1958.

7. Commutative algebra, Vol. II, Van Nostrand, Princeton, N. J., 1961.

Florida State University,

TAllahassee, Florida 